\title{
TITLE:
}

\section{A Series of Collocation Runge-Kutta Methods}

$\operatorname{AUTHOR}(S)$ :

MITSUI, Taketomo; SUGIURA, Hiroshi

CITATION:

MITSUI, Taketomo ... [et al]. A Series of Collocation Runge-Kutta Methods. 数理解析研究所 講究録 1988, 643: 203-225

\section{ISSUE DATE:}

1988-02

URL:

http://hdl.handle.net/2433/100221

RIGHT: 


\section{A Series of Collocation Runge-Kutta Methods}

\section{Taketomo MITSUI and Hiroshi SUGIURA \\ 三井侙友㭅浦洋}

Dept. of Information Eng., Fac. Eng., Nagoya Univ., Nagoya, JAPAN

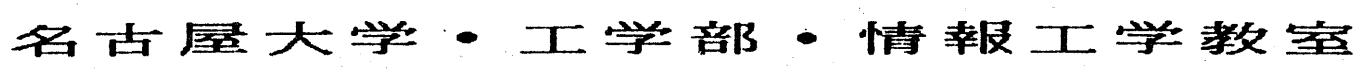

\section{Introduction}

In solving numerical initial value problem of ordinary differential equation

$$
\frac{d y}{d x}=f(x, y) \quad(a<x<b), \quad y(a)=y_{0}
$$

the class of Runge-Kutta formulas (RK formulas, in short) is most important among many discrete variable methods. Especially the stiff problem of (1.1) requires highly sophisticated methods to solve, one of which is the implicit RK formula.

It has the form

$$
y_{n+1}=y_{n}+h \sum_{i=1}^{s} b_{i} f\left(x_{n}+c_{i} h, Y_{i}\right)
$$

where

$$
Y_{i}=y_{n}+h \sum_{j=1}^{s} a_{i j} f\left(x_{n}+c_{j} h, Y_{j}\right) \quad(i=1, \ldots, s) .
$$

Here, the interval of integration, $[a, b]$, is divided by the step-size $h$ so that the step-points

$$
x_{0}(=a)<x_{1}<\ldots<x_{n}<x_{n+1}<\ldots<x_{N}(=b)
$$

are given by

$$
x_{n}=x_{0}+n h
$$


The real parameters $a_{i j}, b_{i}, c_{i}$ specify the method. Formula (1.2), which is called an $s$-stage one, is usually assumed to satisfy

$$
c_{i}=\sum_{j=1}^{\dot{B}} a_{i j} \quad(i=1, \ldots, s) .
$$

This condition can be motivated by the requirement that the approximate solution $y_{n}(n=1,2, \ldots)$ has at least the order one for the exact value $y\left(x_{0}+n h\right)$ with respect to $h$.

To specify the formula, i.e. to determine the parameters $a_{i j}, b_{i}$ and $c_{i}$, the collocation method seems to be most simple and powerful. Although the exact definition will be given in the next section, the sense by the word "collocation" could be explained by considering the polynomial which interpolates the numerical solution $Y_{i}$ in $(1.2 \mathrm{~b})$ at the collocation points $x_{n}$ and $x_{n}+c_{i} h(i=1, \ldots, s)$. In the collocation method one will first choose the dinstinct abscissas $c_{1}, c_{2}, \ldots, c_{8}$, then the other parameters will be uniquely determined. Many known RK formulas, such as Gauss-Legendre, Radau and Lobatto types, belong to this class([2]).

The aim of the present paper is to analyse the process of the choosing the abscissas so as to increase the order of the approximate solution $y_{n+1}$, to investigate the stability of the derived collocation methods, and to reconsider a method proposed by a group of physists ([1]), who called it HIDM (=Higher order Implicit Difference Method). .

\section{The Construction of Collocation RK Method}

\subsection{Collocation Method of Lower Order}

As usual, the local discretization error $\tau\left(x_{n}, h\right)$ of the RK method (1.2) is defined by the following:

$$
\text { (2.1) } \begin{aligned}
\tau\left(x_{n}, h\right) & =\frac{1}{h}\left\{y\left(x_{n+1}\right)-\hat{y}_{n+1}\right\} \\
& =\frac{1}{h}\left\{y\left(x_{n+1}\right)-y\left(x_{n}\right)-h \sum_{i} b_{i} f\left(x_{n}+c_{i} h, \hat{Y}_{i}\right)\right\}
\end{aligned}
$$

where

$$
\hat{Y}_{i}=y\left(x_{n}\right)+h \sum_{j} a_{i j} f\left(x_{n}+c_{i} h, \hat{Y}_{j}\right) \quad(i=1, \ldots, s)
$$


Let us call the RK method to be consistent of order $p$, if $p$ is the largest positive integer such that

$$
\tau\left(x_{n}, h\right)=O\left(h^{p}\right) \text { as } h \downarrow 0
$$

for any sufficiently differentiable solution $y(x)$ of (1.1). Butcher [4] has introduced the simplifying conditions to easily determine the order from very complicated algebraic relations of the parameters.

Definition 1 . An s-stage $R K$ formula is said to satisfy simplifying condition

$$
\begin{aligned}
& A(p) \text { if } \tau\left(x_{n}, h\right)=O\left(h^{p}\right), \\
& B(p) \text { if } \sum_{i=1}^{s} b_{i} c_{i}^{k-1}=\frac{1}{k}(k=1,2, \ldots, p), \\
& C(p) \text { if } \sum_{j=1}^{s} a_{i j} c_{j}^{k-1}=\frac{1}{k} c_{i}^{k} \quad(i=1, \ldots, s, k=1,2, \ldots, p), \\
& D(q) \text { if } \sum_{i=1}^{s} b_{i} c_{i}^{\ell-1} a_{i j}=\frac{1}{\ell} b_{j}\left(1-c_{j}^{\ell}\right) \quad(j=1, \ldots, s, \ell=1,2, \ldots, q), \\
& E(p, q) \text { if } \sum_{i, j=1}^{s} b_{i} c_{i}^{\ell-1} a_{i j} c_{j}^{k-1}=\frac{1}{k(\ell+k)} \quad(\ell=1, \ldots, p, k=1, \ldots, q) .
\end{aligned}
$$

Definition 2 (Burrage,3) . If an s-stage $R K$ formula satisfies the conditions $B(s)$ and $C(s)$, then it is called a collocation method.

The implication of the collocation RK method can be considred as follows.

For the sake of notational convenience, we introduce a scaling for the independent variable by $x=x_{0}+t h$. Then we will consider the differential equation and its approximate solution in the term of the variable $t$. Moreover, for the RK methods, it suffices to consider on the interval $t \in[0,1]$. Hereafter, within the present section, we will restrict ourselves on this situation.

If the RK method (1.2) is regarded as a method of numerical quadrature, we may apply the method to a differential equation depending only on $t$,

$$
\frac{d y}{d t}=h f(t) \quad(0<t<1), y(0)=0 \text {. }
$$


Then it reduces the RK method to the quarature rule

$$
y_{1}=h \sum_{i=1}^{s} b_{i} f\left(c_{i}\right),
$$

where the right-hand side is the approximation of the integral

$$
y(1)=h \int_{0}^{1} f(t) d t
$$

The condition $B(p)$ implies that (2.9) is exact if $f$ is merely a polynomial of $t$ of degree at most $p-1$. This means the quadrature (2.9) is of order $p$.

From (2.8), we have

$$
Y_{i}=h \sum_{j=1}^{s} a_{i j} f\left(c_{j}\right)
$$

for each $i$, which should be considered as an approximation of the integral

$$
y\left(c_{i}\right)=h \int_{0}^{c_{i}} f(t) d t .
$$

Let $P(t)$ be an interpolating polynomial of degree at most $s$. Then the derivative of $P(t)$ is integrated exactly at every internal point $c_{i}$. Thus we obtain from (2.10)

$$
Y_{i}-y_{0}=P\left(c_{i}\right)-P(0)=h \int_{0}^{c_{i}} P^{\prime}(t) d t=h \sum_{j=1}^{s} a_{i j} P^{\prime}\left(c_{j}\right) .
$$

By virtue of $(1.2 b)$, the equation

$$
f\left(c_{j}\right)=P^{\prime}\left(c_{j}\right) \quad(j=1, \ldots, s)
$$

holds, which means that the condition $C(s)$ implies the interpolancy of the derivative $P^{\prime}(t)$ at every internal point $c_{i}(i=1, \ldots, s)$.

In conclusion, the collocation method gives the exact values at $t=1$ for the solution, at $t=c_{i}$ for its derivative if it is a polynomial of degree at most $s$.

Further, a stronger statement holds. Here Butcher made an essential contribution in showing the following 
Theorem 1 . Let an $s$-stage $R K$ formula have distinct abscissas $c_{1}, \ldots$, $c_{s}$ and nonzero weights $b_{1}, \ldots, b_{s}$. Then the following implications hold:

$$
\begin{array}{ll}
(2.11) & A(p) \Rightarrow B(p), \\
(2.12) & A(p+q) \Rightarrow E(p, q), \\
(2.13) & B(p+q) \text { and } C(q) \Rightarrow E(p, q), \\
(2.14) & B(p+q) \text { and } D(p) \Rightarrow E(p, q), \\
(2.15) & B(s+q) \text { and } E(s, q) \Rightarrow C(q), \\
(2.16) & B(p+s) \text { and } E(p, s) \Rightarrow D(p), \\
(2.17) & B(p), C(q) \text { and } D(r) \Rightarrow A(p)(p \leq \min (q+r+1,2 q+2)) .
\end{array}
$$$$
\text { (2.15) } B(s+q) \text { and } E(s, q) \Rightarrow C(q) \text {, }
$$

From (2.17), the collocation RK method of $s$-stage is consistent of order at least $s$ for general initial value problem (1.1) if it has distinct abscissas and nonzero weights. This is easily verified by putting $p=q=s$ and $r=0$ in (2.17). Moreover, it is not difficult to show

Theorem 2 (Dekker-Verwer,2) . Let $s$ distinct abscissas $c_{1}, \ldots, c_{s}$ be given. Then the simplifying conditions $B(s)$ and $C(s)$ uniquely define an $R K$ method with nonzero weights.

Hence, we can focus on the determination of the abscissas for the collocation RK method. Looking back to the interpolating property at the internal point $t=c_{i}$, we have to consider a polynomial on $[0,1]$ of degree $s$ which interpolates the solution $y(t)$. Usually it can be constructed on the fixed interpolating points $\xi_{0}, \xi_{1}, \ldots, \xi_{s}$. Its Lagrange form is given by

$$
L_{s}(t)=\sum_{j=0}^{s} y\left(\xi_{j}\right) \ell_{j}(t)
$$

where

$$
\ell_{j}(t)=\prod_{k \neq j} \frac{t-\xi_{k}}{\xi_{j}-\xi_{k}}
$$

Theorem 3 . For fixed distinct points $\xi_{0}, \ldots, \xi_{s}$ on $[0,1]$, the abscissas $c_{1}$, $\ldots, c_{s}$ of the s-stage collocation method satisfy the equation

$$
\omega_{s}^{\prime}\left(c_{i}\right)=0
$$


where

$$
\omega_{s}(t)=\prod_{j}\left(t-\xi_{j}\right)=\left(t-\xi_{0}\right)\left(t-\xi_{1}\right) \cdots\left(t-\xi_{s}\right) .
$$

Proof will be given by considering the well-known residual formula of the Lagrange interpolating form.

Most conceivable choice for the interpolating points $\xi_{0}, \ldots, \xi_{s}$ on $[0,1]$ is the Newton-Cotes type equidistant distribution

$$
\xi_{j}=\frac{j}{s} \quad(j=0,1, \ldots, s) .
$$

In this case, the determining equation for the abscissas $c_{1}, \ldots, c_{8}$ is given by the algebraic equation

$$
\varphi_{s}^{\prime}(t)=0
$$

where

$$
\varphi_{s}(t)=\prod_{j=0}^{s}\left(t-\frac{j}{s}\right)=t\left(t-\frac{1}{s}\right) \cdots\left(t-\frac{s-1}{s}\right)(t-1) .
$$

It is obvious that the equation (2.21) has $s$ distinct roots on $(0,1)$, and they are situated as $(i-1) / s<c_{i}<i / s(i=1, \ldots, s)$ if they are labelled as $c_{1}<c_{2}<\cdots<c_{8}$.

Since the Newton-Cotes type formula of $s$-stage is a collocation one, it is consistent of order at least $s$. Here we arrive at a question what is the actual order of consistency of the Newton-Cotes type. Before attacking this question, we will state the following Lemma.

Lemma 1 Let $\phi_{p, q}(t)$ be a polynomial of degree $(p+2 q-1)$ defined by

$$
\phi_{p, q}(t)=t^{q}(t-1)^{q} \prod_{j=1}^{p-1}\left(t-\frac{j}{p}\right)
$$

with $p, q \in N$. Then we have

$$
\int_{0}^{1} \phi_{p, q}(t) d t\left\{\begin{array}{lll}
= & 0 & \text { for even } p \\
\neq & 0 & \text { for odd } p
\end{array}\right.
$$


Further, let $\phi_{p, q}^{*}(t)$ be a polynomial of degree $(p+2 q)$ given by

$$
\phi_{p, q}^{*}(t)=t \phi_{p, q}(t)
$$

then we have

$$
\int_{0}^{1} \phi_{p, q}^{*}(t) d t \neq 0
$$

Proof will be carried out through an elementary integral calculus.

Theorem 4 . The s-stage Newton-Cotes type formula is consistent of order $s+1$ for odd $s$, of order $s+2$ for even $s$.

Proof. By virtue of (2.17), it suffices to prove that the condition $B(s+1)$ and $B(s+2)$ are satisfied for the odd case and the even case, respectively.

The condtion $B(s+1)$ means that the Newton-Cotes formula is exact for a polynomial $f(t)$ of degree at most $s$ as the numerical quadraute rule for (2.8). So, let $f(t)$ be a polynomial of degree $s$. Then, in the division of $f(t)$ by $\varphi_{s}^{\prime}(t)$, the quotient and remainder are polynomials of degree 0 and of degree less than $s$, respectively:

$$
f(t)=q \varphi_{s}^{\prime}(t)+r_{s-1}(t) .
$$

Thus

$$
\begin{aligned}
y(1) & =h \int_{0}^{1}\left\{q \varphi_{s}^{\prime}(t)+r_{s-1}(t)\right\} d t \\
& =q h\left[\varphi_{s}(1)-\varphi_{s}(0)\right]+h \int_{0}^{1} r_{s-1}(t) d t \\
& =h \int_{0}^{1} r_{s-1}(t) d t .
\end{aligned}
$$

On the other hand, the identity

$$
\begin{aligned}
\sum_{i=1}^{s} b_{i} f\left(c_{i}\right) & =\sum_{i} b_{i}\left\{q \varphi_{s}^{\prime}\left(c_{i}\right)+r_{s-1}\left(c_{i}\right)\right\} \\
& =\sum_{i} b_{i} r_{s-1}\left(c_{i}\right)
\end{aligned}
$$

holds. The polynomial $r_{s-1}$ is of degree less than $s$ and $c_{1}, \ldots, c_{s}$ are distinct. Thus $\sum b_{i} r_{s-1}\left(c_{i}\right)$ is equal to $\int_{0}^{1} r_{s-1}(t) d t$. 
Next, let $f(t)$ be a polynomial of degree $s+1$. Then we have

$$
f(t)=q(t) \varphi_{s}^{\prime}(t)+r_{s-1}(t)
$$

where $q(t)=q_{1} t+q_{0}$ and $r_{s-1}$ is a polynomial of degree less than $s$. Similar to the above, we can deduce as

$$
\begin{aligned}
y(1) & =h \int_{0}^{1}\left\{q(t) \varphi_{s}^{\prime}(t)+r_{s-1}(t)\right\} d t \\
& =h\left[q(1) \varphi_{s}(1)-q(0) \varphi_{s}(0)\right]-q_{1} h \int_{0}^{1} \varphi_{s}(t) d t+h \int_{0}^{1} r_{s-1}(t) d t \\
& =-q_{1} h \int_{0}^{1} \varphi_{s}(t) d t+h \int_{0}^{1} r_{s-1}(t) d t
\end{aligned}
$$

because $\varphi_{s}(1)=\varphi_{s}(0)=0$.

By virtue of Lemma 1 in the case of $p=s$ and $q=1$, we have

$$
\int_{0}^{1} \varphi_{s}(t) d t\left\{\begin{array}{lll}
= & 0 & \text { for even } p \\
\neq & 0 & \text { for odd } p
\end{array}\right.
$$

This implies that the condition $B(s+2)$ is satisfied for even $s$ while it cannot be satisfied for odd $s$. But if $g(t)$ is of degree 2, i.e. if $f(t)$ is of degree $s+2$, Lemma 1 states

$$
\int_{0}^{1} t \varphi_{s}(t) d t \neq 0
$$

Hence $B(s+3)$ never be satisfied for even $s$.

For some $s$ 's, the graph of $\varphi_{s}(t)$ and $\varphi_{s}^{\prime}(t)$ can be found in Fig. 1. They show the position of the interpolating points $\xi_{0}, \ldots, \xi_{s}$ and the abscissas $c_{1}, \ldots, c_{s}$ of the Newton-Cotes type collocation method.

A physists group ([1]) has presented a discrete variable method for ODE's which can be found to be equivalent to the Newton-Cotes type collocation method. They called it HIDM, which sounds slightly unsuitable. (See Appendix.)

2.2 A Series of Methods of the Same Stage Number 
To increase the order of consistency for the $s$-stage collocation method, we will make a gradual change of the distribution of the interpolating points $\xi_{0}, \ldots, \xi_{s}$ which causes the change of the distribution of the collocation points $c_{1}, \ldots, c_{s}$.

Let $\psi_{k}(t)$ be the polynomial of degree $(s-k)$ given by

$$
\psi_{k}(t)=\left\{\begin{array}{ll}
\prod_{j=1}^{s-k-1}\left(t-\frac{j}{s-k}\right) & \text { for } k=0, \ldots, s-2 \\
1 & \text { for } k=s-1
\end{array} .\right.
$$

Further, define the polynomial $\varphi_{s, k}(t)$ of degree $s+1$ by

$$
\varphi_{s, k}(t)=\frac{d^{k}}{d t^{k}}\left\{t^{k+1}(t-1)^{k+1} \psi_{k}(t)\right\} \quad(k=0,1, \ldots, s-1) .
$$

Note that the identity

$$
\varphi_{s, 0}(t)=\varphi_{s}(t)
$$

holds. Since $t=0$ and $t=1$ are both $(k+1)$-ple root of $t^{k+1}(t-1)^{k+1} \psi_{k}(t)$, $\varphi_{s, k}(0)=\varphi_{s, k}(1)=0$. Obviously $s+1$ distinct real roots of $\varphi_{s, k}(t)=0$ are situated as

$$
\xi_{0}(=0)<\xi_{1}<\cdots<\xi_{s-1}<\xi_{s}(=1)
$$

Thus, $\varphi_{s, k}^{\prime}(t)=0$ can be the determining equation for the abscissas of the collocation method.

Theorem 5 . The s-stage collocation formula determined by the equation

$$
\varphi_{s, k}^{\prime}(t)=0
$$

is consistent of order $s+k+1$ if $s-k$ is odd, and of order $s+k+2$ if $s-k$ is even.

Proof is similar to that of Th.4. Hence it is omitted here.

From Theorems 4 and 5 , we can show the table of increasing the order of consistency in this class. Refer to Table 1. 


\begin{tabular}{|c|rrrrrrr|}
\hline$s \backslash k$ & 0 & 1 & 2 & 3 & 4 & 5 & $\cdots$ \\
\hline 2 & 4 & 4 & & & & & \\
3 & 4 & 6 & 6 & & & & \\
4 & 6 & 6 & 8 & 8 & & & \\
5 & 6 & 8 & 8 & 10 & 10 & & \\
6 & 8 & 8 & 10 & 10 & 12 & 12 & \\
$\vdots$ & & & & & & & $\ddots$ \\
\hline
\end{tabular}

Table 1. The order of consistency for $(s, k)$.

Theorem 6 . The s-stage collocation method determined by the equation

$$
\varphi_{s, s-1}^{\prime}(t)=0
$$

is nothing but the s-stage Gauss-Legendre method.

This theorem can be proved through the Rodrigues' formula for the Legendre polynomials.

In Fig.2, one can see the change of the zeros for the polynomial

$$
\frac{d^{\ell}}{d t^{\ell}}\left\{t^{k+1}(t-1)^{k+1} \psi_{k}(t)\right\} \quad(\ell=0,1, \ldots, k+1)
$$

for some pairs of $(s, k)$.

On closing the present section, we have established a way to generate a series of $s$-stage collocation method starting from the Newton-Cotes type $((s+1)$-st or $(s+2)$-nd order) up to the Gauss-Legendre formula (2s-th order), increasing the order of consistency two by two.

\section{A-Stability of the Collocation RK Method}

\subsection{Derivation of the Stability Factor}

For the consideration of the stability of RK method, the matrix and vector notations make the process simple and elegant. Let $A$ and $b$ be the matrix and the vector given by

$$
A=\left(a_{i j}\right) \quad(1 \leq i, j \leq s) \text { and } b=\left(b_{1}, \ldots, b_{s}\right)^{T} \text {, }
$$


respectively. Defining the vector $e$ all of whose components are equal to unity, the stability factor $R(z)$ of the RK method (1.2) is given by

$$
R(z)=\frac{\operatorname{det}\left(I-z A+z e b^{T}\right)}{\operatorname{det}(I-z A)},
$$

which can be found in many literatures (e.g. Dekker-Verwer[2]).

In the $s$-stage collocation method, $A$ and $b$ are uniquely determined by the set of the abscissas $c_{1}, \ldots, c_{s}$ provided that they are distinct (Th.2). Thus, let us define the polynomial

$$
\pi_{s}(t)=\prod_{j=1}^{s}\left(t-c_{j}\right)=\left(t-c_{1}\right)\left(t-c_{2}\right) \cdots\left(t-c_{s}\right),
$$

which is the derivative of $\omega_{s}(t)$ in Th.3 divided by its leading coefficient to make it monic. Thus we obtain

$$
\pi_{s}(t)=t^{s}+\sum_{j=0}^{s-1} p_{j} t^{j}
$$

where the coefficients $p_{j} \quad(j=0, \ldots, s-1)$ can be represented by the fundamental symmetric functions of $c_{1}, \ldots, c_{s}$.

We introduce the Vandermonde matrix

$$
V=\left[\begin{array}{cccc}
1 & c_{1} & \cdots & c_{1}^{s-1} \\
1 & c_{2} & \cdots & c_{2}^{s-1} \\
\vdots & \vdots & & \vdots \\
1 & c_{s} & \cdots & c_{s}^{s-1}
\end{array}\right]
$$

and the diagonal matrices

$$
\begin{aligned}
& C=\operatorname{diag}\left[c_{1}, c_{2}, \cdots, c_{s}\right], \\
& S=\operatorname{diag}\left[1, \frac{1}{2}, \cdots, \frac{1}{s}\right] .
\end{aligned}
$$

Then, the simplifying conditions $B(s)$ and $C(s)$ are equivalent to the matrix identities given by the following (Dekker-Verwer[2]):

$$
B(s) \quad: \quad b^{T} V=e^{T} S,
$$




$$
C(s) \quad: \quad A V=C V S .
$$

The direct calculation leads to the equation

$$
C V S=\left[c_{i}^{j} / j\right]
$$

On the other hand, the identity

$$
\begin{aligned}
& {\left[1, c_{i}, \cdots, c_{i}^{s-1}\right]\left[\begin{array}{cccccc}
0 & & & & & -p_{0} / s \\
1 & 0 & & & & -p_{1} / s \\
& 1 / 2 & \ddots & & & \vdots \\
& & \ddots & \ddots & & \vdots \\
& & & \ddots & 0 & -p_{s-2} / s \\
& & & & 1 /(s-1) & -p_{s-1} / s
\end{array}\right]} \\
& =\left[c_{i}, \frac{c_{i}^{2}}{2}, \cdots, \frac{c_{i}^{s-1}}{s-1},-\frac{\sum_{j=0}^{s-1} p_{j} c_{i}^{j}}{s}\right]
\end{aligned}
$$

holds. By virtue of (3.3), the right-hand side of the above identity is equal to the $i$-th row vector of the matrix product $C V S$. Hence, we can establish the equation

$$
V^{-1} C V S=\left[\begin{array}{cccccc}
0 & & & & & -p_{0} / s \\
1 & 0 & & & & -p_{1} / s \\
& \frac{1}{2} & \ddots & & & \vdots \\
& & & \ddots & & \vdots \\
& & & & 0 & -p_{s-2} / s \\
& & & & \frac{1}{s-1} & -p_{s-1} / s
\end{array}\right]
$$

Let $W$ be the diagonal matrix given by

$$
W=\operatorname{diag}[1,1,2 !, 3 !, \cdots,(s-1) !] .
$$


From (3.8),(3.9) and (3.10); we obtain

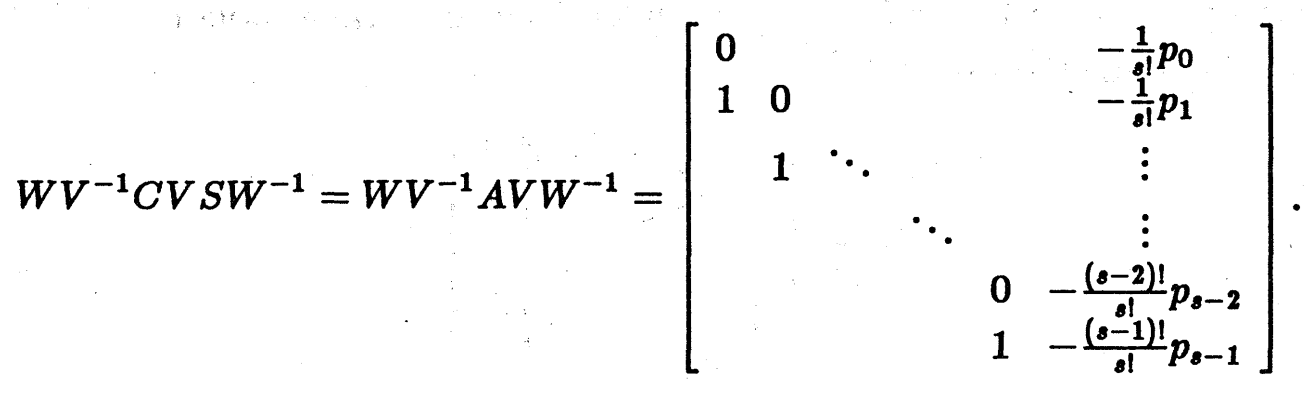

The right-hand side matrix is the companion matrix for the polynomial

$$
d(z)=\frac{p_{0}}{s !}+\frac{p_{1}}{s !} z+\cdots+\frac{(s-1) ! p_{s-1}}{s !} z^{s-1}+z^{s}=\frac{1}{s !} \sum_{j=0}^{s} j ! p_{j} z^{j}
$$

That is, the identity

$$
d(z)=\operatorname{det}\left(z I-W V^{-1} A V W^{-1}\right)
$$

holds. Hence, we arrive at the following lemma.

Lemma 2 . The denominator polynomial $\Delta(z)$ of the stability factor $R(z)$ is given by

$$
\Delta(z)=z^{8} d\left(\frac{1}{z}\right)
$$

Proof. By calculation, we have

$$
\begin{aligned}
\Delta(z) & =\operatorname{det}(I-z A)=z^{8} \operatorname{det}\left(\frac{1}{z} I-A\right) \\
& =z^{8} \operatorname{det}\left(\frac{1}{z} I-W V^{-1} A V W^{-1}\right)=z^{8} d\left(\frac{1}{z}\right) \cdot \square
\end{aligned}
$$

Next, we will consider the numerator of $R(z)$. The numerator $N(z)$ is given by $N(z)=\operatorname{det}\left(I-z A+z e b^{T}\right)$. From (3.7) and (3.8), we obtain $e b^{T}=e e^{T} S V^{-1}$ and $A=C V S V^{-1}$, which implies

$$
A-e b^{T}=\left(C V-e e^{T}\right) S V^{-1}
$$


The $(i, j)$-th component of the matrix $C V-e e^{T}$ is given by $c_{i}^{j}-1$. Then, putting $\gamma_{i}=c_{i}-1(i=1, \ldots, s)$, we introduce the Vandermonde matrix $U$ and the diagonal matrix $\Gamma$ by

$$
U=\left[\begin{array}{cccc}
1 & \gamma_{1} & \cdots & \gamma_{1}^{s-1} \\
1 & \gamma_{2} & \cdots & \gamma_{2}^{s-1} \\
\vdots & \vdots & & \vdots \\
1 & \gamma_{s} & \cdots & \gamma_{s}^{s-1}
\end{array}\right]
$$

$$
\Gamma=\operatorname{diag}\left[\gamma_{1}, \gamma_{2}, \cdots, \gamma_{s}\right]
$$

Taking the identity

$$
\gamma_{i}^{j}=\left(c_{i}-1\right)^{j}=\sum_{\ell=0}^{j}(-1)^{j-\ell}\left(\begin{array}{l}
j \\
\ell
\end{array}\right) c_{i}^{\ell}
$$

into the consideration, the two matrices $V$ and $U$ are in the linear relation. In fact, we obtain

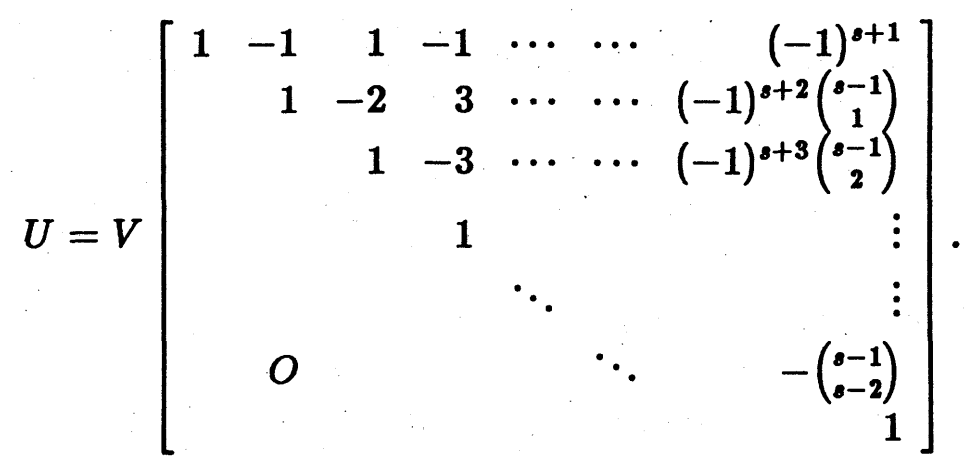

On the other hand,

$$
\left(C V-e e^{T}\right) S=\left[\begin{array}{cccc}
c_{1}-1 & \left(c_{1}^{2}-1\right) / 2 & \cdots & \left(c_{1}^{s}-1\right) / s \\
\vdots & \vdots & & \vdots \\
\vdots & \vdots & & \vdots \\
c_{s}-1 & \left(c_{s}^{2}-1\right) / 2 & \cdots & \left(c_{s}^{s}-1\right) / s
\end{array}\right]
$$


Thus, from (3.17) we have

$$
\begin{aligned}
\left(C V-e e^{T}\right) S V^{-1} U & =\left[\begin{array}{cccc}
\gamma_{1} & \gamma_{1}^{2} / 2 & \cdots & \gamma_{1}^{s} / s \\
\vdots & \vdots & & \vdots \\
\gamma_{b} & \gamma_{s}^{2} / 2 & \cdots & \gamma_{s}^{s} / s
\end{array}\right] \\
& =\Gamma U S,
\end{aligned}
$$

which, with (3.14), implies the identity

$$
\left(A-e b^{T}\right) U=\Gamma U S .
$$

Hence, similar to the denominator case, we can establish the following result. By the shift of the variable $t$ in $\pi_{s}(t)$, let us define the polynomial $\rho_{s}(t)$ by

$$
\begin{aligned}
\rho_{s}(t) & =\pi_{s}(t+1) \\
& =t^{s}+\sum_{j=0}^{s-1} r_{j} t^{j}
\end{aligned}
$$

where $r_{j}(j=0, \ldots, s-1)$ is the coefficient.Further, introducing the polynomial

$$
e(z)=\frac{r_{0}}{s !}+\frac{r_{1}}{s !} z+\cdots+\frac{(s-1) ! r_{s-1}}{s !} z^{s-1}+z^{s}=\frac{1}{s !} \sum_{j=0}^{s} j ! r_{j} z^{j}
$$

we have the following

Lemma 3 . The numerator polynomial $N(z)$ of the stability factor $R(z)$ is given by

$$
N(z)=z^{s} e\left(\frac{1}{z}\right)
$$

Due to the above Lemmas, $R(z)$ is represented by

$$
R(z)=\frac{N(z)}{\Delta(z)}
$$

where $\Delta(z)$ and $N(z)$ are given by (3.13) and (3.22), respectively. The maximum magnitude principle implies the necessary and sufficient conditions $(S 1)$ and $(S 2)$ for the A-stability of the collocation RK method as follows: 
(S1) All the roots of $\Delta(z)$ belong to the right half-plane of the complex $\boldsymbol{z}$. by $i$.)

(S2) $|N(i y)| \leq|\Delta(i y)|$ for every real $y$. (The imaginary unit is denoted

Definition 3 If the distribution of the abscissas $c_{1}, c_{2}, \ldots, c_{8}$ is symmetric with respect to $1 / 2$, the collocation $R K$ method of these abscissas is called as symmetric.

Theorem 7 If the collocation method is symmetric, it is A-stable iff all the zeros of $\Delta(z)$ are in the right half-plane.

\subsection{A-Stability of the Newton-Cotes Type Methods}

In the case of the Newton-Cotes type method, the collocation points are given by (2.21). The polynomial $\pi_{s}(t)$ is then given by

$$
\pi_{s}(t)=\frac{1}{s+1} \varphi_{s}^{\prime}(t)
$$

where $\varphi_{s}(t)$ is defined by (2.22). Utilizing Lemma 2, we can calculate the denominator polynomials $\Delta(z)$ for various number of $s$ 's. By Theorem 7, we are to investigate the zeros of $\Delta(z)$ whether all of them are situated in the right half-plane. In other words, the question is whether all of the zeros of $\Delta(-z)$ are in the left half-plane $C^{-}$. The symbolic and algebraic computation by computer gives the polynomials $\Delta(-z)$ as in Table 2. As can be seen, all the coefficiets of one polynomial have the same sign with the leading coefficient. Thus they satisfy the necessary condition for the required root distribution. But, as is well known, the exact solution is only possible by e.g. the Routh-Hurwitz criteria.

Given a polynomial $f(z)$ as

$$
f(z)=f_{0} z^{n}+f_{1} z^{n-1}+\cdots+f_{n-1} z+f_{n},
$$

where $f_{0}>0$, then the Routh-Hurwitz criteria first defines the following $n$-square matrix $Q$. 


$$
Q=\left[\begin{array}{ccccc}
f_{1} & f_{3} & f_{5} & \cdots & f_{2 n-1} \\
f_{0} & f_{2} & f_{4} & \cdots & f_{2 n-2} \\
0 & f_{1} & f_{3} & \cdots & f_{2 n-3} \\
0 & f_{0} & f_{2} & \cdots & f_{2 n-4} \\
\vdots & \vdots & \vdots & & \vdots \\
\vdots & \vdots & \vdots & & \vdots
\end{array}\right]
$$

Here $f_{j}=0$ for $j>n$. The necessary and sufficient condition for all the roots of $f(z)$ being in $C^{-}$is that all the principal minors of $Q$ are positive.

We have calculated the matrix $Q$ and its principal minors for every $\Delta(-z)$, whose results are shown in Table 3. This leads us to the following.

Theorem 8 The Newton-Cotes type collocation methods whose stage number is less than 9 are $A$-stable.

Table 2. $\Delta(-z)$ for the Newton-Cotes type formulas

$$
\begin{aligned}
\Delta_{2}(-z)= & z^{2}+6 z+12 \\
\Delta_{3}(-z)= & z^{3}+11 z^{2}+54 z+108 \\
\Delta_{4}(-z)= & 3 z^{4}+50 z^{3}+420 z^{2}+1920 z+3840 \\
\Delta_{5}(-z)= & 12 z^{5}+274 z^{4}+3375 z^{3}+25500 z^{2}+112500 z+225000 \\
\Delta_{6}(-z)= & 5 z^{6}+147 z^{5}+2436 z^{4}+26460 z^{3}+189000 z^{2}+816480 z+1632960 \\
\Delta_{7}(-z)= & 30 z^{7}+1089 z^{6}+22981 z^{5}+331681 z^{4}+3361400 z^{3}+23193660 z^{2} \\
& +98825160 z+197650320 \\
\Delta_{8}(-z)= & 105 z^{8}+4566 z^{7}+118124 z^{6}+2153088 z^{5}+28734720 z^{4} \\
& +278691840 z^{3}+1878589440 z^{2}+7927234560 z+15854469120 \\
\Delta_{9}(-z)= & 560 z^{9}+28516 z^{8}+879525 z^{7}+19539360 z^{6}+327229875 z^{5} \\
& +4151341530 z^{4}+39060913500 z^{3}+258918055200 z^{2} \\
& +1084777369200 z+2169554738400
\end{aligned}
$$


Table 3. Principal minors of the Newton-Cotes type formulas

\begin{tabular}{|c|rrrrrrr}
\hline$k \backslash s$ & 2 & 3 & 4 & 5 & 6 & 7 \\
\hline 1 & 6 & 11 & 50 & 274 & 147 & 1089 \\
2 & 72 & 486 & 15240 & 618750 & 225792 & $1.50 \cdots \times 10^{7}$ \\
3 & & 52488 & $1.96 \cdots \times 10^{7}$ & $8.07 \cdots \times 10^{9}$ & $2.49 \cdots \times 10^{9}$ & $1.77 \cdots \times 10^{12}$ \\
4 & & & $7.54 \cdots \times 10^{10}$ & $5.14 \cdots \times 10^{14}$ & $1.72 \cdots \times 10^{14}$ & $1.51 \cdots \times 10^{18}$ \\
5 & & & & & $1.15 \cdots \times 10^{20}$ & $6.90 \cdots \times 10^{19}$ & $9.42 \cdots \times 10^{24}$ \\
6 & & & & & & $1.12 \cdots \times 10^{26}$ & $4.04 \cdots \times 10^{32}$ \\
7 & & & & & & $7.99 \cdots \times 10^{40}$ \\
8 & & & & & & \\
9 & & & & & & \\
\hline
\end{tabular}

\begin{tabular}{rr|}
\hline 8 & 9 \\
\hline 4566 & 28516 \\
$3.13 \cdots \times 10^{8}$ & $1.41 \cdots \times 10^{10}$ \\
$2.09 \cdots \times 10^{14}$ & $7.64 \cdots \times 10^{16}$ \\
$1.10 \cdots \times 10^{21}$ & $3.38 \cdots \times 10^{24}$ \\
$4.48 \cdots \times 10^{28}$ & $9.29 \cdots \times 10^{32}$ \\
$1.60 \cdots \times 10^{37}$ & $2.58 \cdots \times 10^{41}$ \\
$4.93 \cdots \times 10^{46}$ & $-1.01 \cdots \times 10^{53}$ \\
$7.81 \cdots \times 10^{56}$ & $-5.01 \cdots \times 10^{64}$ \\
& $-1.08 \cdots \times 10^{77}$ \\
\hline
\end{tabular}

Appendix is omitted because of the limit of space. Hence interested reader will be given the full manuscript on his request by the authors.

\section{References}

[1] Abe,K., Ishida,A., Watanabe,T., Kanada, Y. \& Nishikawa,K., HIDM - New numerical method for differntial equations, Kakuyugo-kenkyu, 57(1987), 85-95 (in Japanese).

[2] Dekker,K. \& Verwer, J.G., Stability of Runge-Kutta Methods for Stiff Nonlinear Differntial Equations, North-Holland, 1984.

[3] Burrage, K., High order algebraically stable Runge-Kutta methods, $B I T, 18(1978), 373-383$.

[4] Butcher, J.C., Implicit Runge-Kutta processes, Math. Comp., 18(1964), 50-64. 
Fig.1 The graphs of $\varphi_{s}(t)$ and $\varphi_{s}^{\prime}(t)$.

Fig.1.1 The case of $s=3$.
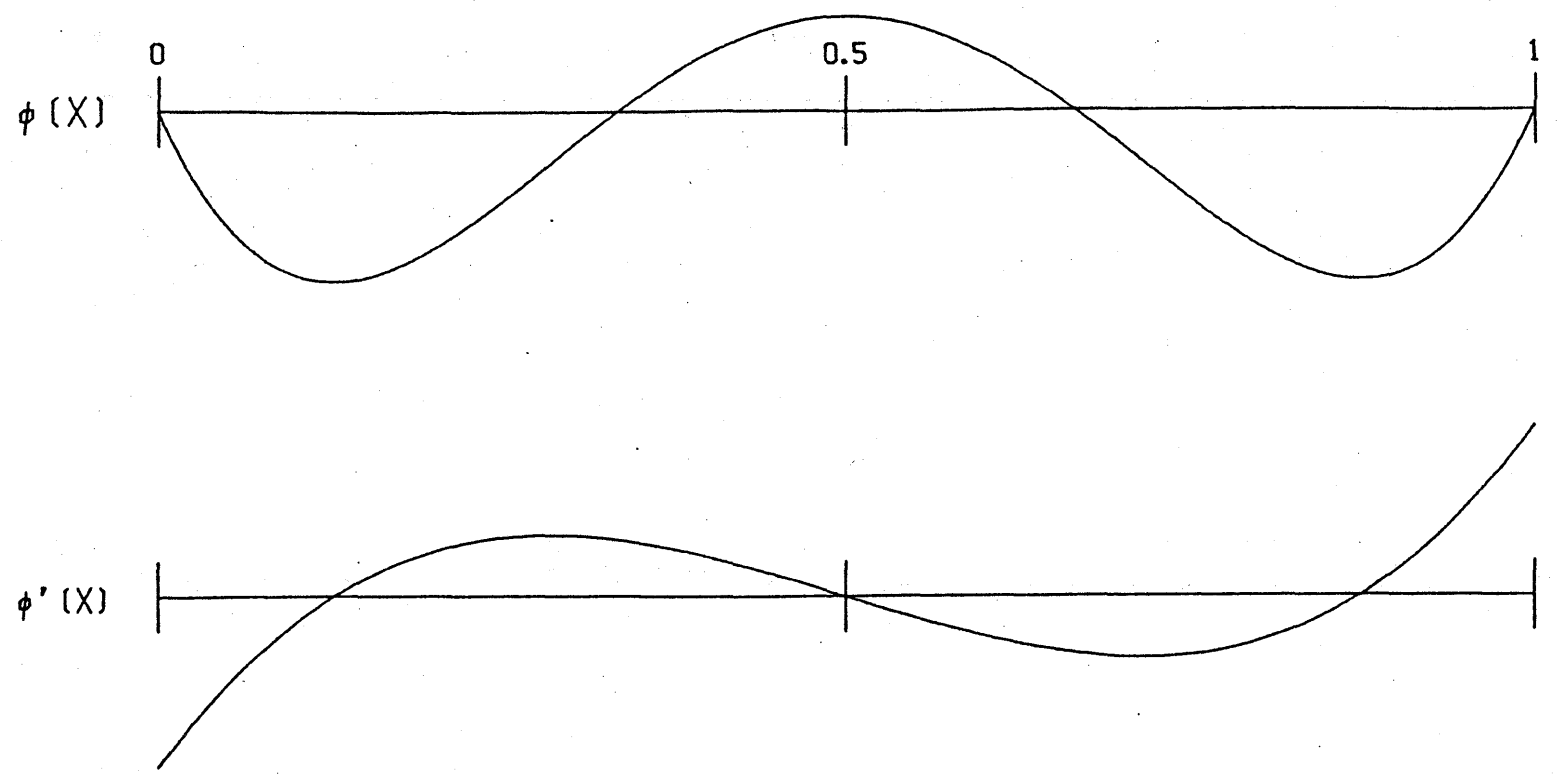

Fig.1.2 The case of $s=4$.
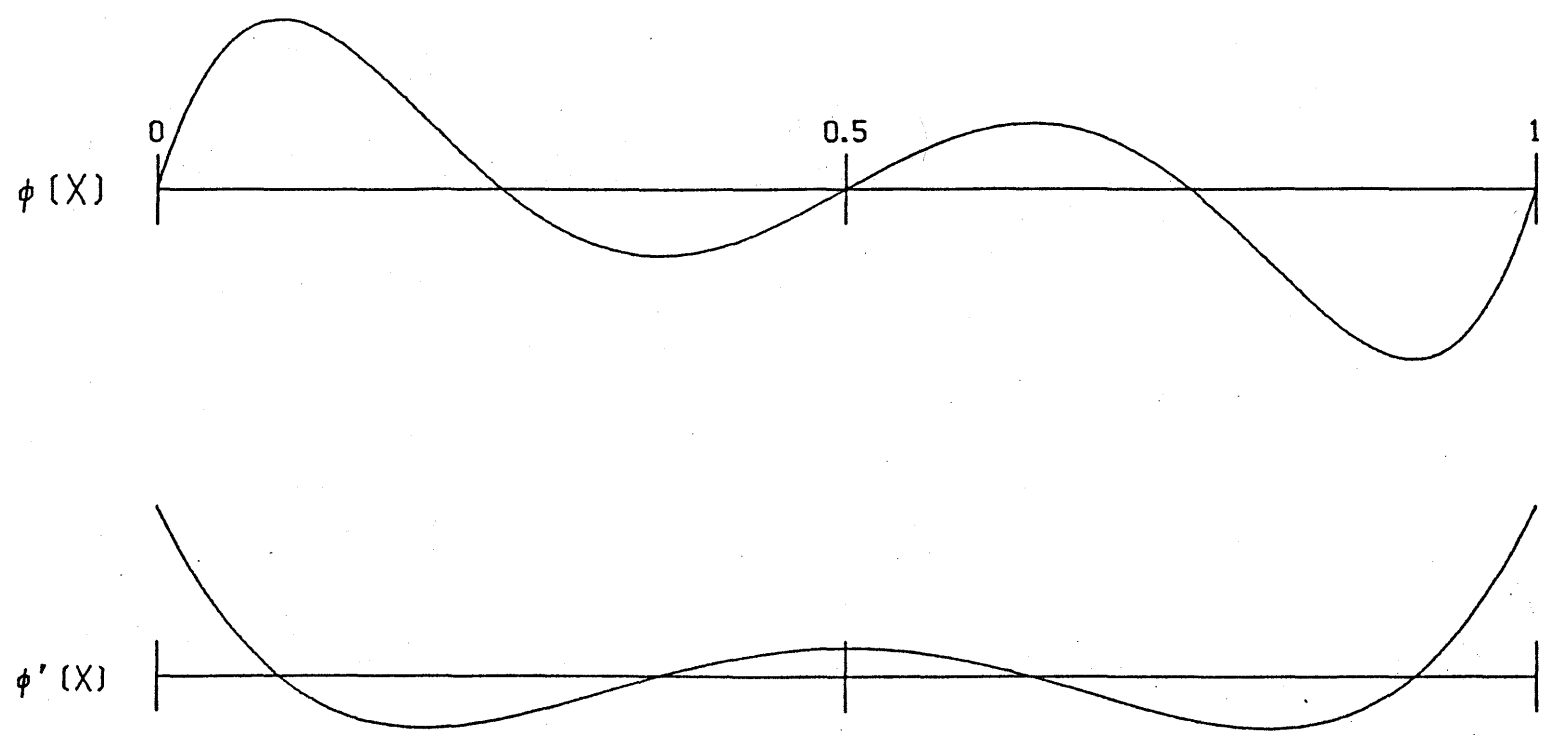
Fig.1.3 The case of $s=5$.
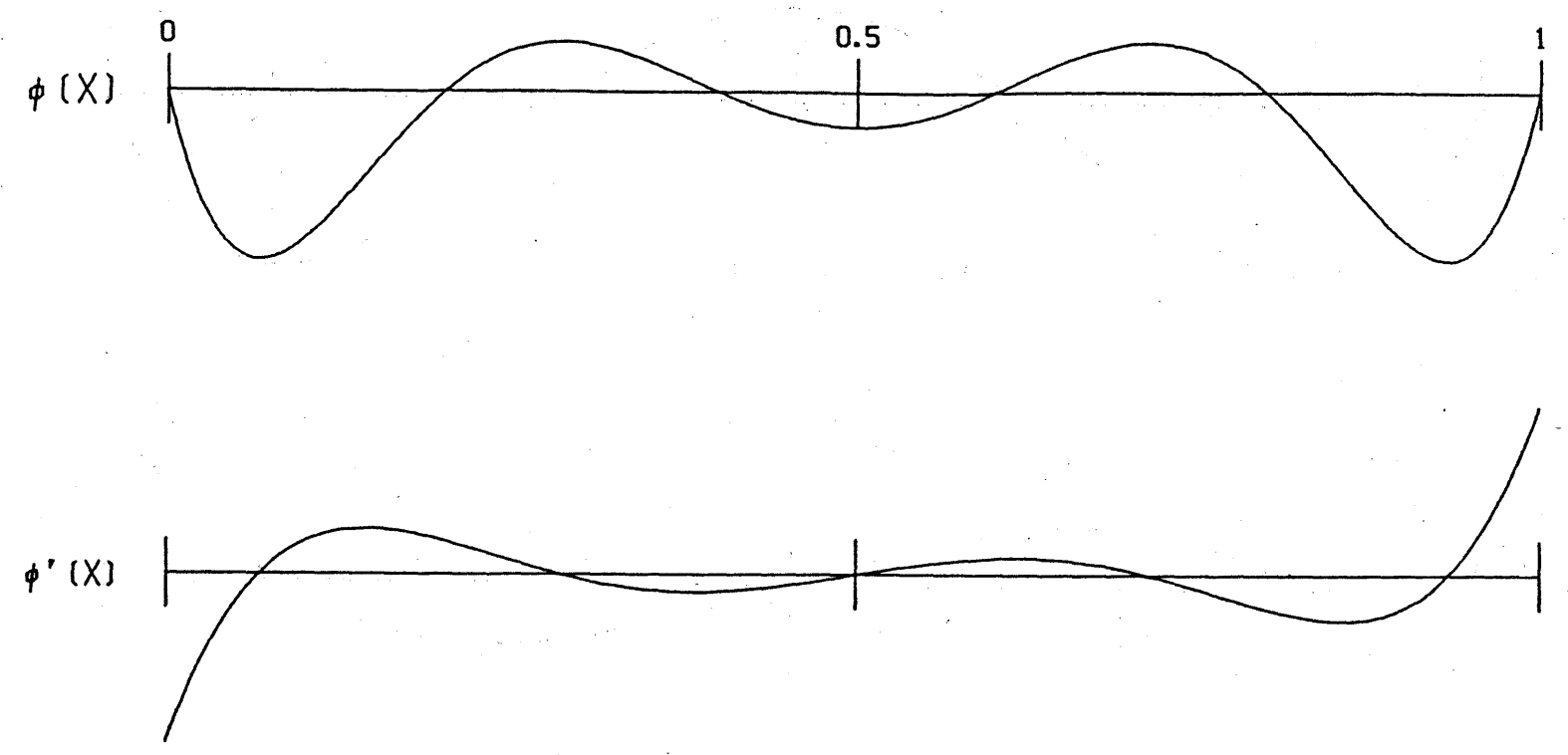

Fig.1.4 The case of $s=6$.
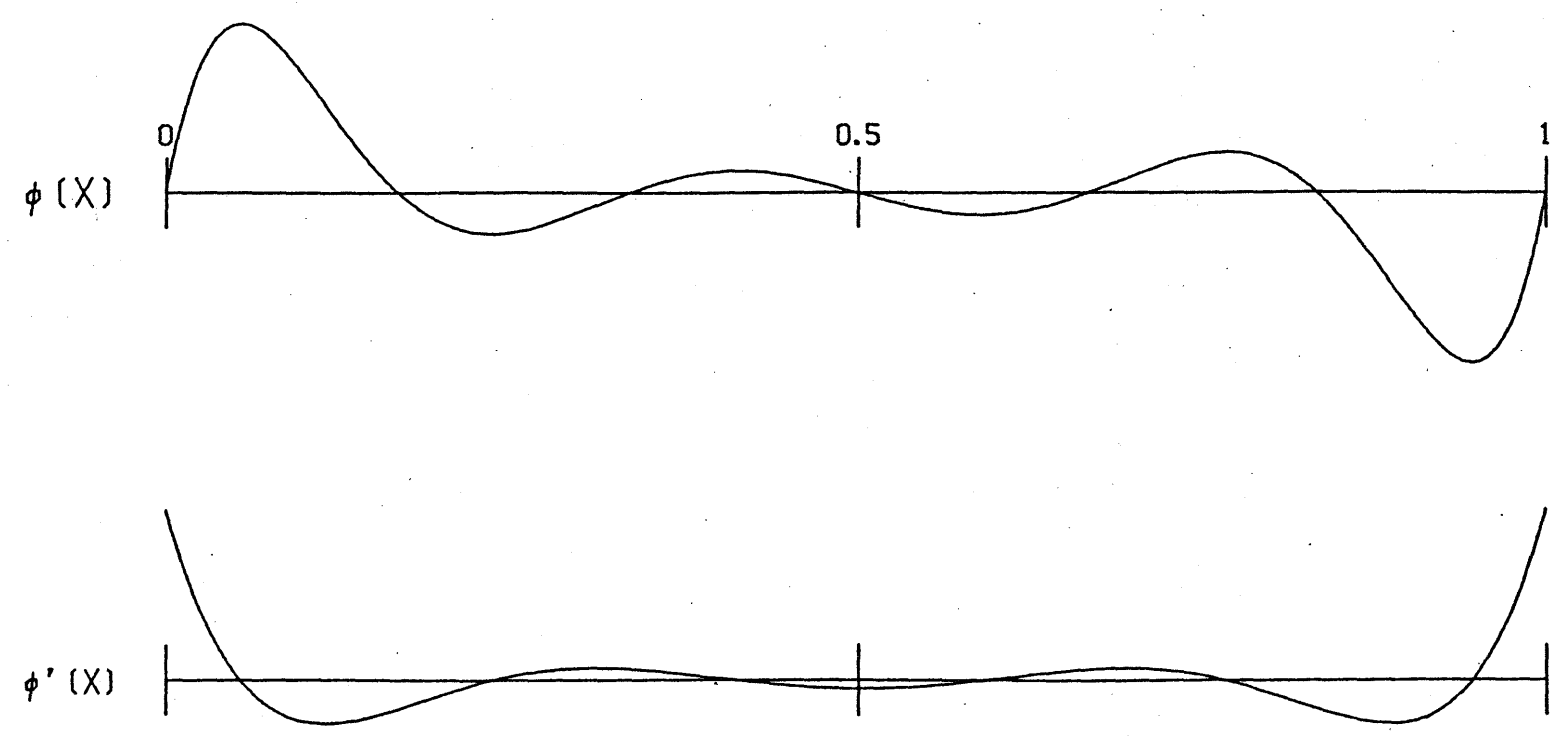
Fig.2 The zeros of the polynomials $\frac{d^{\ell}}{d t^{\ell}}\left\{t^{k+1}(t-1)^{k+1} \psi_{k}(t)\right\}$.

Fig.2.1 The case of $k=0 \quad(\ell=0,1)$.
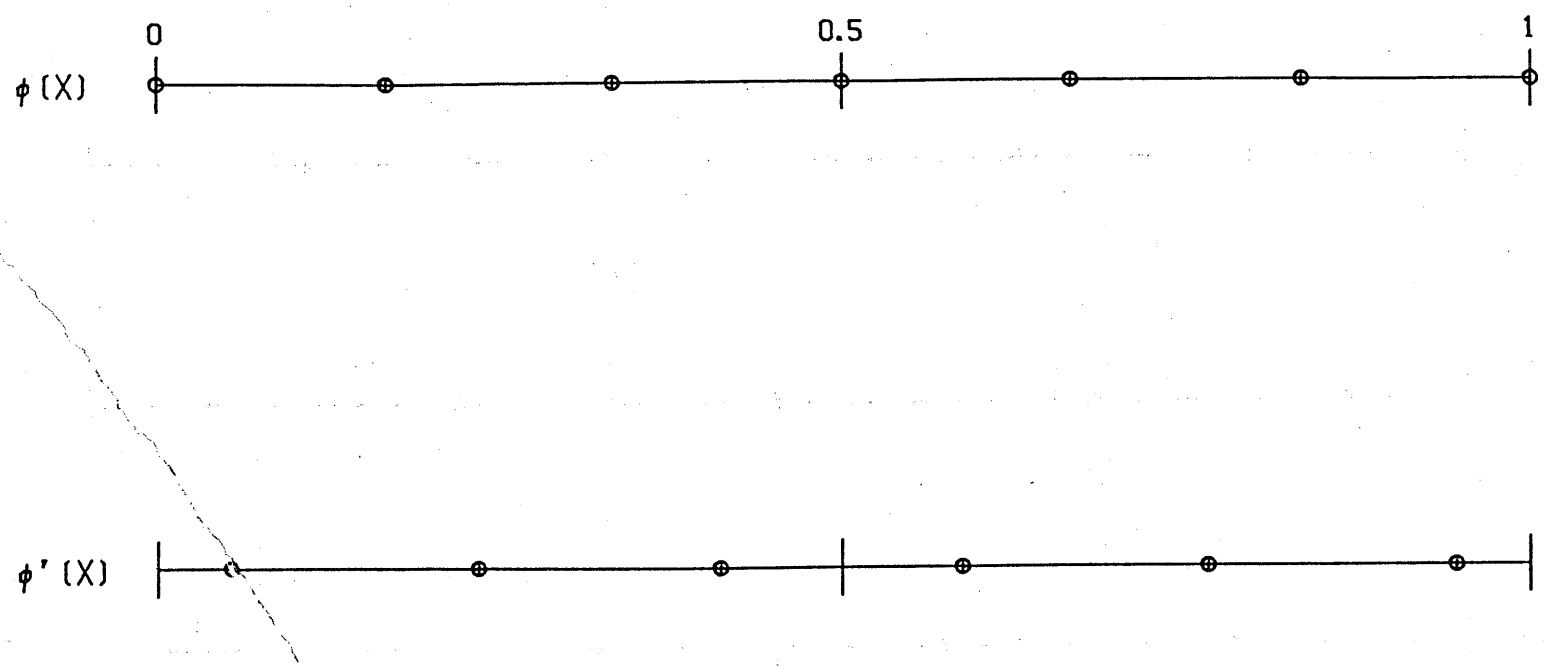

Fig.2.2 The case of $k=1 \quad(\ell=0,1,2)$.

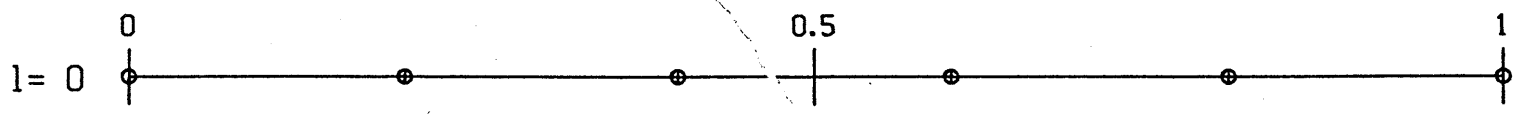

$\phi(X)$

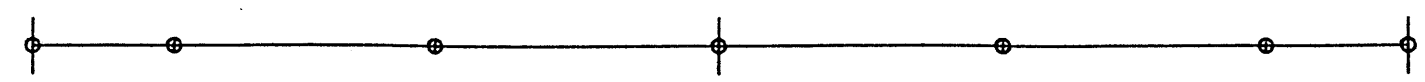

$\phi^{\prime}(X)$
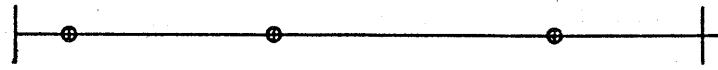
Fig.2.3 The case of $k=2 \quad(\ell=0,1,2,3)$.
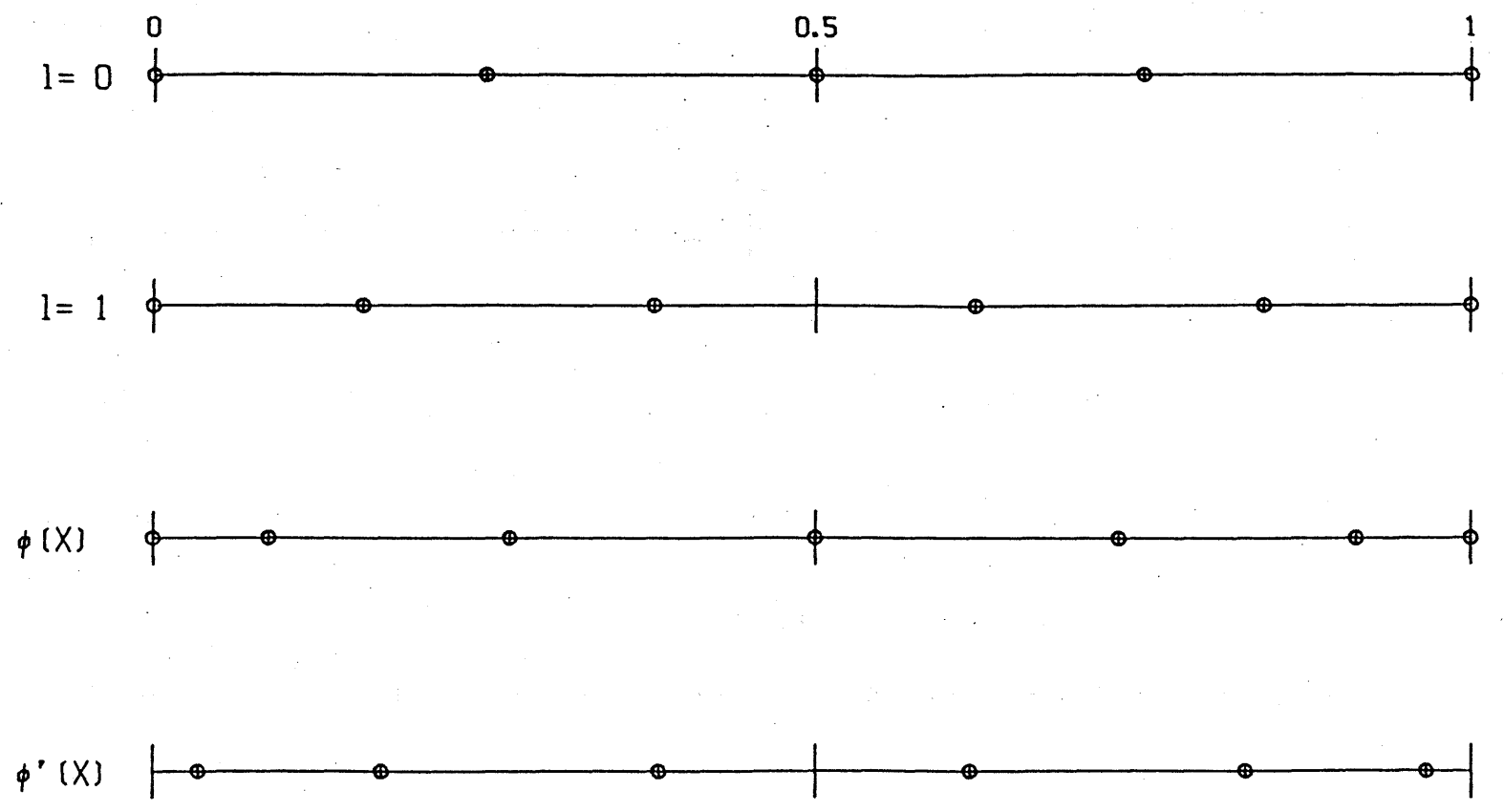

Fig.2.4 The case of $k=3 \quad(\ell=0,1, \ldots, 4)$.
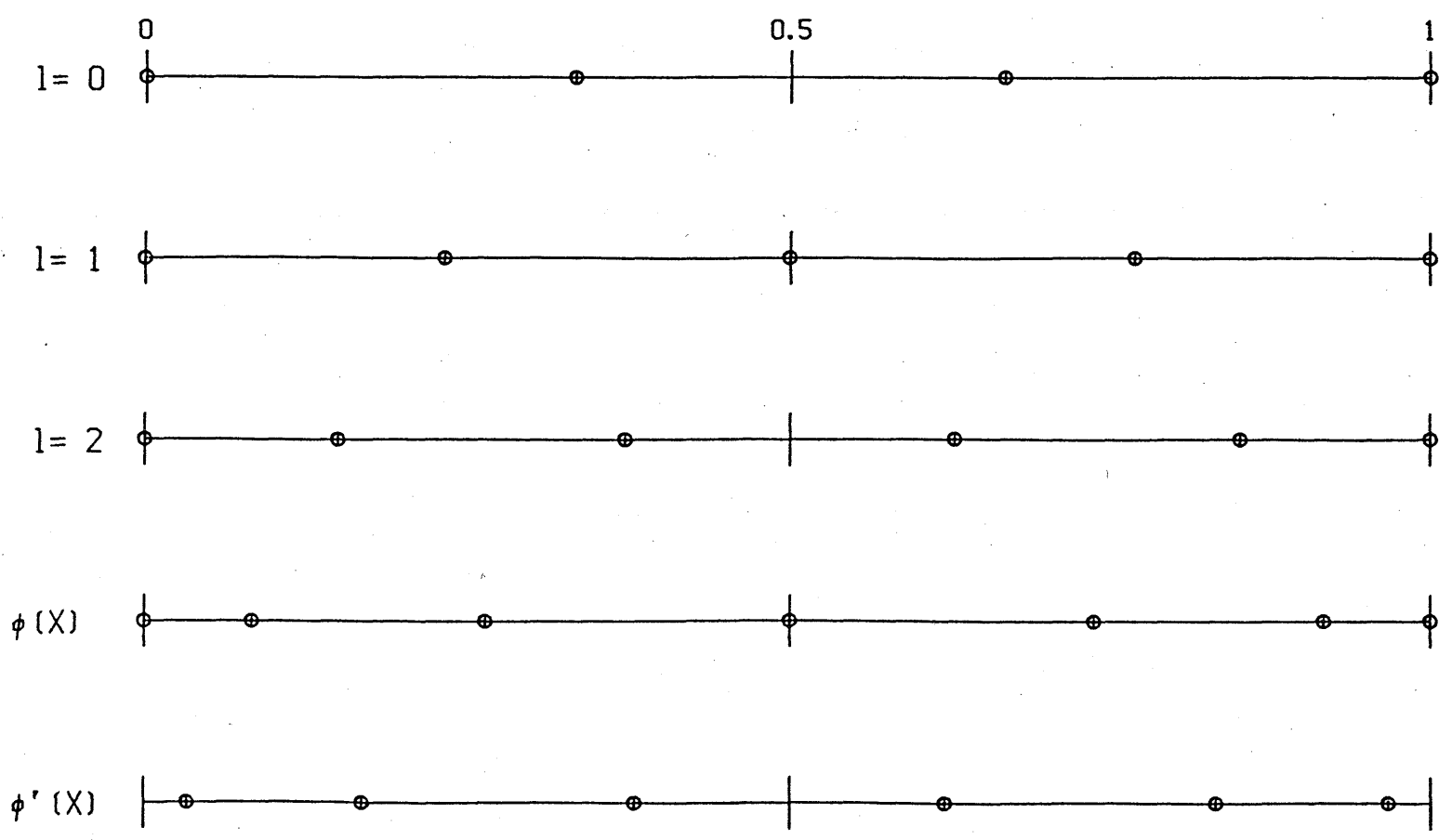
Fig.2.5 The case of $k=4 \quad(\ell=0,1, \ldots, 5)$.

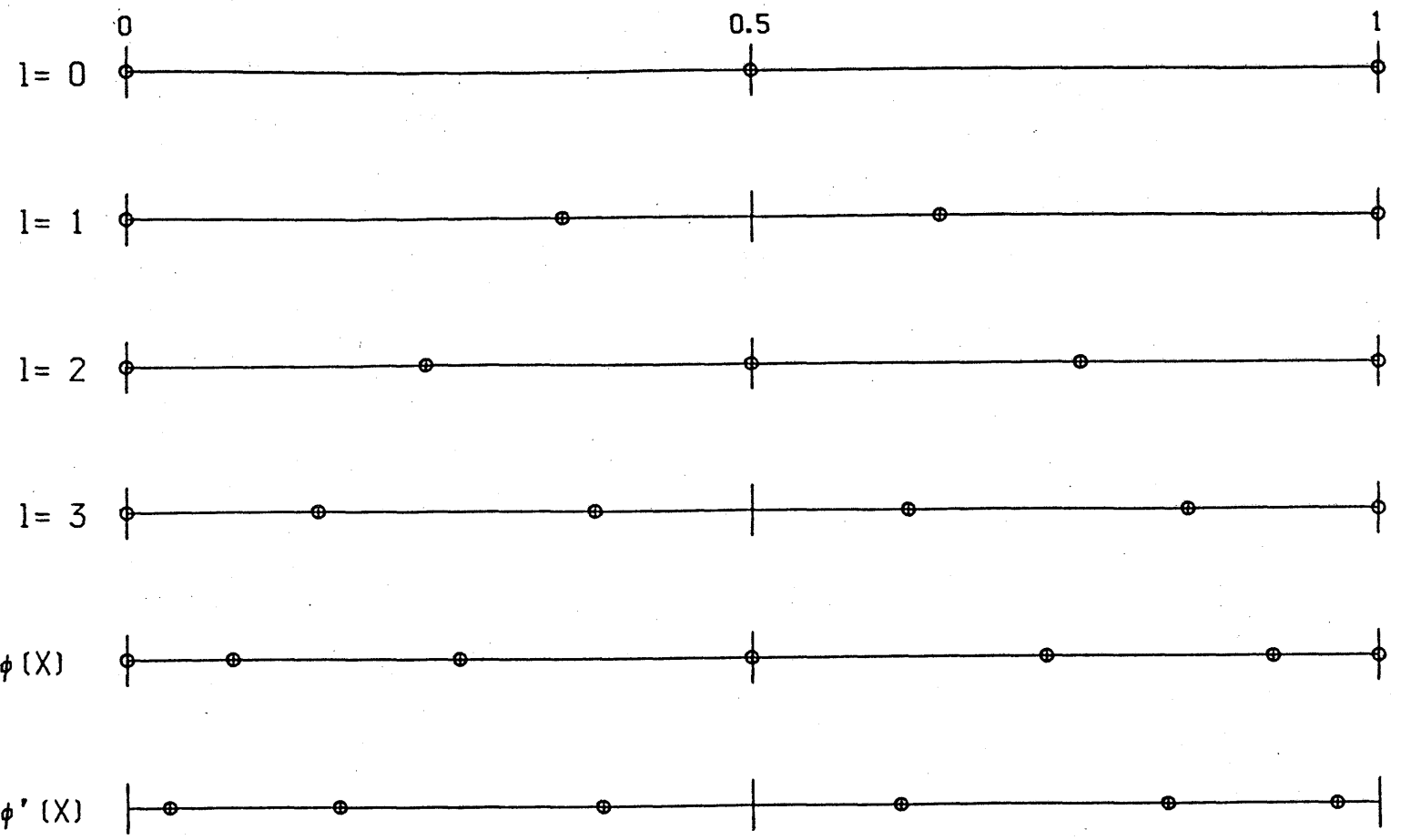

Fig.2.6 The case of $k=5 \quad(\ell=0,1, \ldots, 6)$.

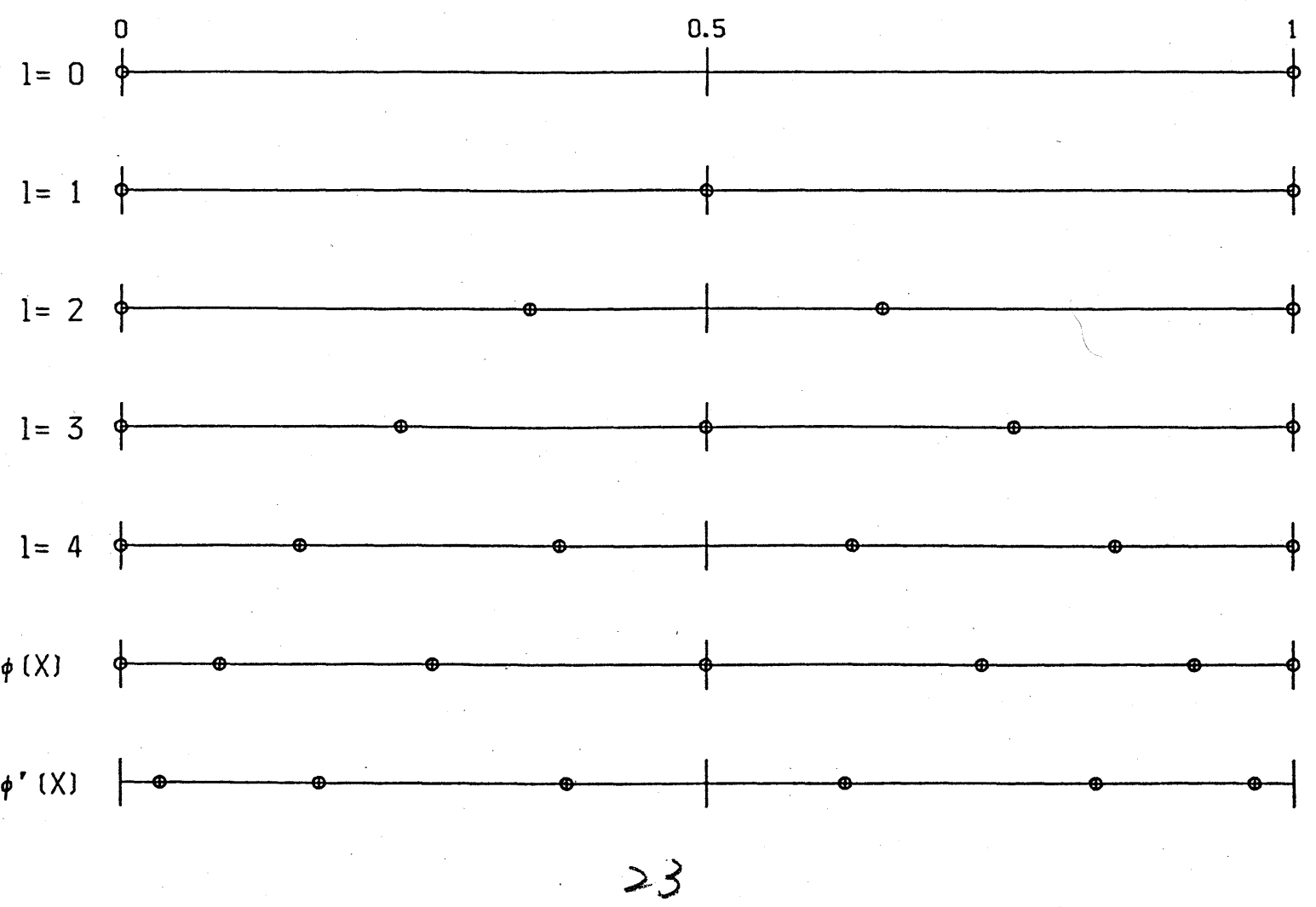

